\title{
Path Planning of Five - section scurve Interpolation Method
}

\author{
Boquan Zhang ${ }^{\text {a) }}$ and Sheng Gao \\ School of computers, Guangdong University of Technology, Guangzhou 510003, China \\ a) Corresponding author: 1244818453@qq.com
}

\begin{abstract}
In the ROS environment, study the D H modeling, trajectory planning algorithm and simulation of Youbot mechanical arm. In the ROS environment, with MoveIt!Tools, mechanical arm Yobot model based on D H parameter model, the calculation of the mechanical arm homogeneous transformation matrix, through simulation toolbox is inverse kinematics simulation and motion simulation of trajectory planning control research. The displacement, velocity, acceleration and terminal trajectory of each joint of the manipulator were obtained by simulation. From the simulation results, it can be seen that the research method is of great practical significance to the research on the motion control of the manipulator, which provides reference for the research of the manipulator control and optimization algorithm of Youbot.
\end{abstract}

Key words: ROS; Motion control; Trajectory planning; Five - section scurvy interpolation method.

\section{INTRODUCTION}

The mechanical arm has a wide range of applications in industrial production, such as the use of mechanical arms to carry goods, assembly products, etc. One of the most common problems in mechanical arm technology is the high precision trajectory planning, which is also the most important part in the research of mechanical arm technology. Traditional method of manipulator trajectory planning problems need according to each specific structure, in line with the actual situation of the control system, involving the end trajectory planning algorithm, the kinematics inverse solution, interference and constraints and joints fine interpolation process, a process that workload big, time consuming, difficult to widely used in high efficiency of modern society. Nowadays, the rapid development of robot software development platform provides a new way to solve this technical problem [1].

Based on the full application of robot manipulator trajectory planning problems convenience features and achievements of open source software development platform [2], and on this basis, through the secondary development, the five sections s deceleration curve interpolation method, is of great significance to control the robot arm.

\section{ALGORITHM DESCRIPTION}

Based on the five-polynomial interpolation method, the five-section S curve [4] and deceleration interpolation method have more detailed planning on the trajectory and can adapt to more complicated work requirements. In the 5 $\mathrm{S}$ deceleration curve interpolation method, the whole exercise increased the requirements of the uniform motion track, make the whole movement process more conform to the actual working status of general, is helpful to extend the service life of mechanical arm.

The five-segment $\mathrm{S}$ curve plus deceleration interpolation method is divided into five segments: acceleration, deceleration, constant speed, deceleration and deceleration. Set in addition to the uniform section of thought, the remaining four periods of time is equal to Ta, a total of T, uniform speed for VS, four variable slope sizes for K, L, the whole period of trajectory of the total displacement and acceleration segment displacement L1, deceleration segment displacement $\mathrm{L} 2$. The following relationships are: 


$$
\left\{\begin{array}{l}
\mathrm{T}_{a}=\sqrt{\frac{V_{s}}{K}} \\
L_{1}=\frac{1}{6} K T_{a}^{3} \\
L_{2}=\frac{1}{6} K T_{a}^{3} \\
T=4 T_{a}+\frac{L-2 L_{1}-2 L_{2}}{V_{s}}
\end{array}\right.
$$

The acceleration segmentation function is as follows:

$$
\mathrm{a}=\left\{\begin{array}{l}
K t,\left(0 \leq t \leq T_{a}\right) \\
-K\left(t-2 T_{a}\right),\left(T_{a} \leq t \leq 2 T_{a}\right) \\
0,\left(2 T_{a} \leq t \leq T-2 T_{a}\right) \\
-K\left[t-\left(T-2 T_{a}\right)\right],\left(T-2 T_{a} \leq t \leq T-T_{a}\right) \\
K(t-T),\left(T-T_{a} \leq t \leq T\right)
\end{array}\right.
$$

Because the velocity function is used to get the acceleration function, then the acceleration is integral and satisfies the endpoint constraint condition, then the velocity segment function:

$$
v=\left\{\begin{array}{l}
\frac{1}{2} K t^{2},\left(0 \leq t \leq T_{a}\right) \\
-\frac{1}{2} K\left(t-2 T_{a}\right)^{2}+A T_{a}^{2},\left(T_{a} \leq t \leq 2 T_{a}\right) \\
V_{s},\left(2 T_{a} \leq t \leq T-2 T_{a}\right) \\
-\frac{1}{2} K\left[t-\left(T-2 T_{a}\right)\right]^{2}+K T_{a}^{2},\left(T-2 T_{a} \leq t \leq T-T_{a}\right) \\
\frac{1}{2} K(t-T)^{2},\left(T-T_{a} \leq t \leq T\right)
\end{array}\right.
$$

According to the mathematical knowledge, the Angle function can be obtained by integrating the speed and satisfying the constraint conditions of the two endpoints.

$$
S=\left\{\begin{array}{l}
\frac{1}{6} K t^{3},\left(0 \leq t \leq T_{a}\right) \\
-\frac{1}{6} K\left(t-2 T_{a}\right)^{3}+K T_{a}^{2} t-K T_{a}^{3},\left(T_{a} \leq t \leq 2 T_{a}\right) \\
K T_{a}^{2} t-K T_{a}^{3}\left(2 T_{a} \leq t \leq T-2 T_{a}\right) \\
-\frac{1}{6} K\left[t-\left(T-2 T_{a}\right)\right]^{3}+K T_{a}^{2} t-K T_{a}^{2},\left(T-2 T_{a} \leq t \leq T-T_{a}\right) \\
\frac{1}{2} K(t-T)^{3}-2 K T_{a}^{3}+K T_{a}^{2} T,\left(T-T_{a} \leq t \leq T\right)
\end{array}\right.
$$




\section{ALGORITHM SIMULATION RESULTS}

First starting point and goal at the end of a given mechanical arm posture, the $5 \mathrm{~s}$ deceleration curve interpolation method is adopted to calculate the solution of the inverse kinematics geometry algorithm, except for uniform time of the rest of the four equal to $\mathrm{Ta}$, a total of $\mathrm{T}$, uniform velocity $\mathrm{v}$, four variable speed section of the slope $\mathrm{K}$ size, the whole period of trajectory as input, the total displacement of L and MATLAB programming involved in the process of mechanical arm movement joint trajectory, angular velocity and angular acceleration time curve as well as the mechanical arm end actuator displacement simulation. Can be seen from the simulation results, after picking the whole mechanical arm motion, the angular velocity of movement joint - time curve, angular acceleration - time curve is continuous smooth, without acceleration curve of mutation. This indicates that the mechanical arm runs smoothly and does not have an impact phenomenon and fully meets its motion requirements.

The displacement, velocity and acceleration of the joint in the simulation experiment are shown as follows:

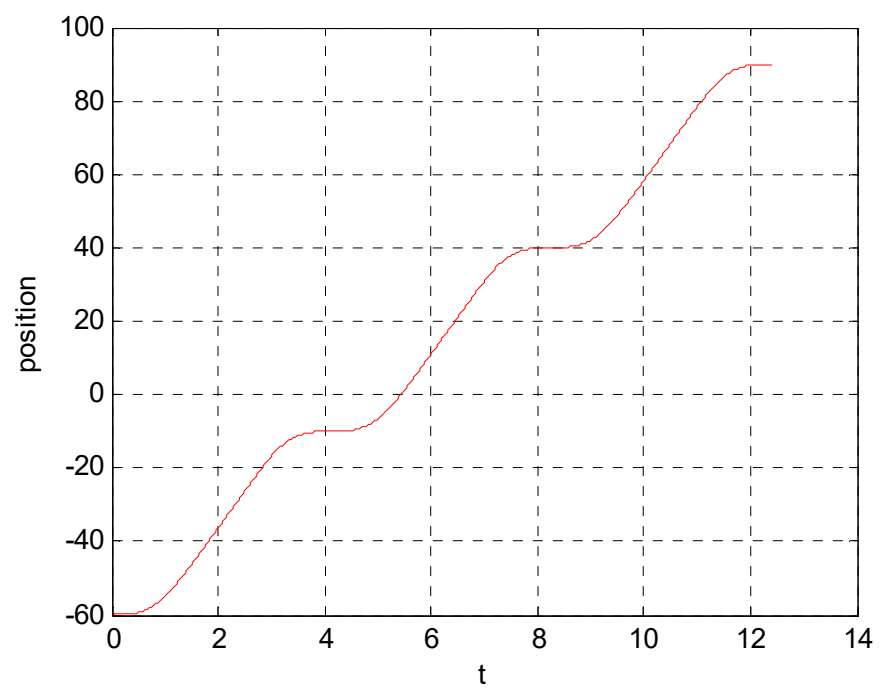

FIG 1. position- $\mathrm{t}$

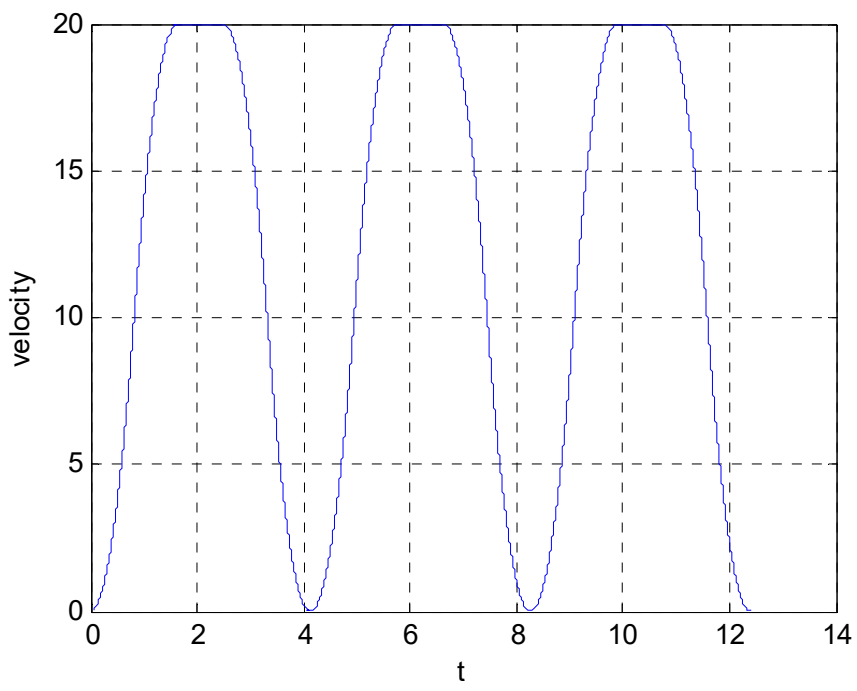

FIG 2. velocity- $\mathrm{t}$ 


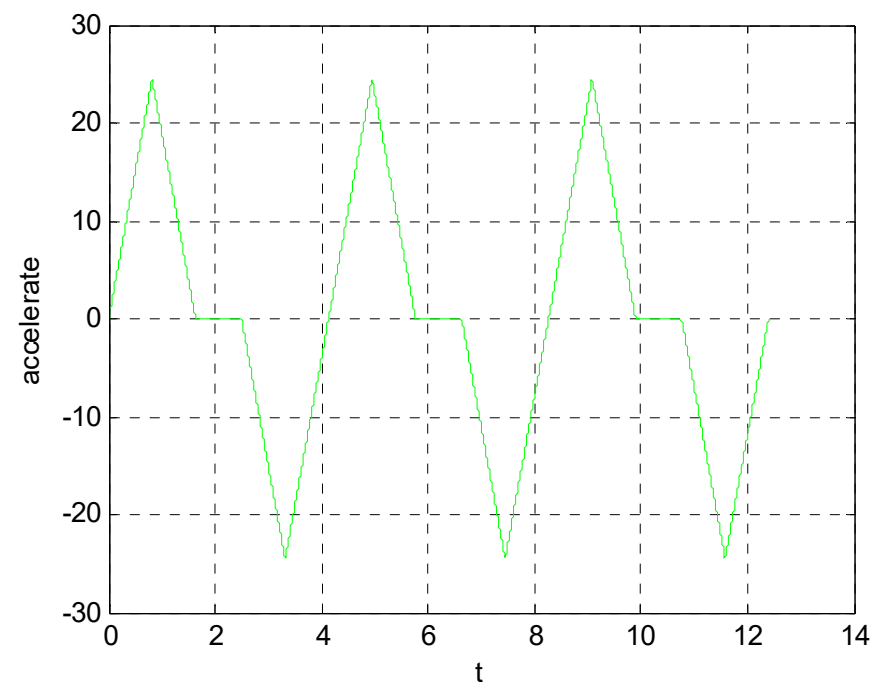

FIG 3. accelerate-t

According to the figure of displacement, velocity and acceleration and time, the displacement curve is a smooth curve during the whole motion, and the slope of the curve changes periodically over time. And the velocity curve is a periodic curve that looks like a $\mathrm{n}$, and the whole thing is a period of change from acceleration to constant speed and deceleration. The corresponding acceleration curve is starting from 0 speed first, to the maximum attenuation, start with the same slope and keep for a while to 0 into uniform phase, and then entered the stage of accelerated reduction finally entered the stage of slowdown in the same slope, then end the whole motion process. The acceleration and deceleration control algorithm of s-type curve is continuous, and the speed curve is smooth, and it is effective to overcome the disadvantages of linear interpolation and exponential addition and deceleration.

On the macroscopic analysis, the whole process of displacement, velocity and acceleration curve accords with the general law of mechanical arm movement is homework, satisfies the given all the constraints, and each parameter mutation do not exist, benefit to decrease the damage of the mechanical arm parts, extend the life of the machine.

\section{CONCLUSION}

In this paper, the five-segment s-curve algorithm is studied, which provides a theoretical basis for the acceleration and deceleration of the step motor. According to the comparison results, the five-paragraph s-curve acceleration and deceleration method can overcome the problems caused by the traditional method of adding and subtracting, which is more suitable for the current high-speed control system.

\section{REFERENCES}

1. Jun Sun, Jialiang Zhang, Ling Ma. Dobot Simulation and trajectory planning algorithm of mechanical arm. [J]. Machinery and Electron, 2016, 34(6):72-75.

2. Rujia Liu, ang Wang, Qiang Zhang. Study on the trajectory planning of robot arm based on ROS. [J]. Navigation and Positioning and Timing., 2016, 3(6).

3. Xiujuan Zheng. Study on motion control and trajectory planning algorithm of mobile manipulator. [D]. Wuhan university of science and technology, 2012.

4. Yongchao Ma. The study of a five-paragraph s-curve acceleration and deceleration method. [J]. Industrial control computer, 2014(12):60-61.

5. Xiaowei Ni, Jianqun Liu, ongwei Xu. Study on the new algorithm of decelerating five-segment $\mathrm{S}$ curve acceleration and deceleration. [J]. Machine tool and hydraulic, 2016, 44(17):93-97. 